\title{
Additives for the Stabilization of Double- Stranded DNA in UV-MALDI MS
}

\author{
Anne M. Distler and John Allison \\ Department of Chemistry, Michigan University, East Lansing, Michigan, USA
}

Molecular complexes such as double-stranded oligonucleotides contain non-covalent bonds that are difficult to maintain in the MALDI experiment. Quantifiers are introduced in order to evaluate, summarize, and compare spectra from experiments in which additives are used to stabilize duplex oligonucleotides. Compounds known to complex with and stabilize duplex molecules can be useful as additives in MALDI. Spermine and methylene blue, present at concentrations similar to the matrix, are detected, bound to the duplex. When peptides are used as additives, the duplex is stabilized when the peptide is present at an amount less than that of the duplex. (J Am Soc Mass Spectrom 2002, 13, 1129-1137) (C) 2002 American Society for Mass Spectrometry

$\mathrm{U}$ ltraviolet matrix-assisted laser desorption/ionization mass spectrometry (UV MALDI MS) [1] has become an important technique for the analysis of biomolecules such as peptides and oligonucleotides. While analysis of oligonucleotides initially lagged behind peptide analysis, the development of new matrices and matrix additives has made significant improvements in oligonucleotide characterization by MALDI MS [2-7]. The first additives used with oligonucleotides were ammonium salts such as ammonium hydrogen citrate [8] and ammonium fluoride $[9,10]$. It is assumed that the additive alters the charge state or environment in which the analyte exists in the target crystals. In addition to the elimination of multiple alkali-ion adduction, the ammonium salt seems to play a significant role in enhancing both the desorption and the ionization of intact oligonucleotides [9]. Recently, other additives have been developed for the MALDI analysis of oligonucleotides, notably the tetraamine spermine [2, 3], related amines [11], and fucose [4, 5].

Through the use of additives, UV MALDI MS has been used successfully to study many types of covalent molecules. However, molecular complexes containing non-covalent bonds are more difficult to analyze in the UV MALDI experiment. Using a technique such as UV MALDI MS, a double-stranded oligonucleotide can be desorbed and ionized as a singly charged, gas phase ion, although only a few examples have been reported [12-14]. While oligonucleotides used in this study are 12-14 bases in length, these duplexes can serve as models for DNA/drug binding studies. Duplexes of low molecular weight are particularly useful because

Published online August 19, 2002

Address reprint requests to Dr. J. Allison, Chemistry Department, Michigan State University, East Lansing, MI 48824, USA. E-mail: allison@cem. msu.edu the resolution in a MALDI-time-of-flight (TOF) MS experiment is sufficient to detect small, organic molecules bound to the duplex. Also, they represent an analytical challenge of mass spectrometry, representative of non-covalent complexes, for which techniques could be developed to stabilize them.

When a double-stranded oligonucleotide, $\mathrm{M}_{1} \mathrm{M}_{2}$, is analyzed in negative-ion MALDI-TOF MS from a simple matrix/analyte target, only ions representing the two single strands, $\left(\mathrm{M}_{1}-\mathrm{H}\right)^{-}$and $\left(\mathrm{M}_{2}-\mathrm{H}\right)^{-}$, are usually detected [15]. Since $\mathrm{M}_{1} \mathrm{M}_{2}$ is not detected as $\left(\mathrm{M}_{1} \mathrm{M}_{2}-\mathrm{H}\right)^{-}$or $\left(\mathrm{M}_{1} \mathrm{M}_{2}+\mathrm{H}\right)^{+}$in UV MALDI MS, the duplex must dissociate at some point before acceleration and detection of the ions. There are many steps in the MALDI experiment where the double-stranded species could denature including the initial formation of the MALDI target and the desorption/ionization $(\mathrm{D} / \mathrm{I})$ process. The presence of stabilizing additives in the experiment may maintain the non-covalent complex throughout all phases of the experiment.

There are several compounds known to complex with and stabilize double-stranded DNA at the cellular level. In cells, DNA is negatively-charged due to the ionization of the phosphate groups in the phosphodiester backbone. The phosphate groups can be affiliated with counter-ions such as sodium ions, magnesium ions, and polyamines such as spermine [16, 17]. The polyamines are positively charged at physiological $\mathrm{pH}$ and bind to DNA, shielding the negative charges and decreasing the repulsion between the strands. Spermine allows double-stranded DNA to condense into a more compact structure in cells [18]. When oligonucleotides are crystallized for X-ray crystallographic analysis, spermine is frequently added to facilitate crystal growth $[18,19]$. Spermine may also stabilize the duplex in the MALDI experiment.

While not naturally found in cells, several small, 
organic compounds are known to bind to the major and minor grooves of double-stranded oligonucleotides or intercalate between the bases. The presence of such species may stabilize the duplex through the MALDI crystal growth and desorption/ionization processes. One example is ethidium bromide. Ethidium (Et) is a positively-charged species that is known to intercalate between the bases of duplexes. Ethidium bromide has been found to stabilize the double-stranded duplex poly(dT) bound to poly $(\mathrm{dA})$, increasing the melting temperature by $14{ }^{\circ} \mathrm{C}[20,21]$.

While certain small molecules are known to stabilize double-stranded DNA, larger molecules such as proteins and peptides may also have a stabilizing effect on duplexes. Interactions between DNA and proteins play important roles in many biochemical processes. Chromosomes contain DNA and an equal mass of histone and non-histone proteins [22]. Histones, molecular weights between 13,000 and 30,000 g/mol, are highly conserved across species and contain many basic residues. These positively-charged amino acid side chains interact with and stabilize the DNA through salt bridges.

Similar to histones, protamines are arginine-rich proteins found in sperm cells. Cationic protamines bind to DNA with their $\alpha$-helices in the major groove of the DNA, where they enable the tight packing of the duplexes [23]. While the main driving forces for protamine-DNA binding are the ionic interactions, there is a distinct cooperativity that cannot be explained on the basis of electrostatic interactions alone [24]. Protamines perform functions similar to histones, but are generally smaller, with molecular weights between 4000 and $10,000 \mathrm{~g} / \mathrm{mol}$ [25]. Non-covalent complexes between proteins and oligonucleotides have been studied in UV MALDI previously [26]. However, the effect of the presence of peptides on the spectra of double-stranded oligonucleotides has not been examined.

There is no new matrix or additive that allows for the complete conservation of the intact duplex in UV MALDI MS. For this reason, insights must be extracted from the smaller spectral changes that can be measured when the experimental variables are changed. We introduce here three simple quantifiers that allow for UV MALDI spectra of double-stranded oligonucleotides to be evaluated, summarized, and compared. Using these quantifiers, we demonstrate that the presence of stabilizing additives has a measurable effect on the duplex region of the resulting UV MALDI mass spectra. The quantifiers allow one to maintain a numerical focus on the goals in these measurements, possibly leading to the development of a blended matrix (with multiple additives), in which components each serve to chemically address different aspects of this system.

\section{Experimental}

The double-stranded oligonucleotides used in this work were purchased from the Michigan State University
Table 1. Oligonucleotides used in this work

\begin{tabular}{|c|c|c|c|}
\hline Name & Sequence & $\begin{array}{c}\text { Average } \\
\text { molecular } \\
\text { weight }\end{array}$ & $T_{m}{ }^{a}$ \\
\hline \multirow[t]{2}{*}{ Duplex 1} & 5'-CCGGAATTGGCC-3' & 3646 & \\
\hline & 3'-GGCCTTAACCGGTT-5' & 4254 & $40^{\circ} \mathrm{C}$ \\
\hline \multirow[t]{2}{*}{ Duplex 2} & 5'-АСССАСССАССС-3' & 3480 & \\
\hline & 3'-TGGGTGGGTGGG-5' & 3813 & $42^{\circ} \mathrm{C}$ \\
\hline \multirow[t]{2}{*}{ Duplex 3} & 5'-AAAAACCAAAAA-3' & 3648 & \\
\hline & 3'-TTTTTGGTTTTT-5' & 3638 & $28^{\circ} \mathrm{C}$ \\
\hline
\end{tabular}

${ }^{\mathrm{a}} \mathrm{T}_{\mathrm{m}}$ is the melting temperature at which the duplex dissociates.

Macromolecular Structure, Sequencing, and Synthesis Facility (East Lansing, MI) and are shown in Table 1, with their molecular weights and melting temperatures $\left(\mathrm{T}_{\mathrm{m}}\right)$. They are all stable in solution at room temperature with melting temperatures ranging from 28 to $42{ }^{\circ} \mathrm{C}$. The presence of the duplex in the initial solutions was confirmed by UV analysis at $259 \mathrm{~nm}$.

The peptides, dynorphin A, fibrinogen binding inhibitor peptide (FBI peptide), $\beta$-melanocyte stimulating hormone $(\beta-\mathrm{MSH})$, katacalcin, kemptide, trilysine, $\mathrm{N}-\varepsilon$ acetyl-lysine, leucine enkephalin, Boc-MNF-amide, hexaalanine, trialanine methyl ester, and triserine were all purchased from Sigma (St. Louis, MO) and used without further purification. Peptides were dissolved in MilliQ water at concentrations of $1.0 \mathrm{pmol} / \mu \mathrm{L}$.

The stock duplex solutions had concentrations of 25 $\mathrm{pmol} / \mu \mathrm{L}$. The compounds, 6-aza-2-thiothymine (ATT), chromomycin, daunomycin, ethidium bromide, methylene blue, distamycin, and Hoechst 33258 were purchased from Aldrich Inc. (Milwaukee, WI). Spermine (sp) and 3-hydroxypicolinic acid (HPA) were purchased from Fluka (Milwaukee, WI). When spermine was used as a matrix additive, it was prepared at a concentration of $25 \mathrm{mM}$ in water. The stock solutions of ethidium bromide and methylene blue were $1 \mathrm{mM}$ in water. Saturated matrix solutions were made using a 1:1 acetonitrile/spermine solution or 1:1 acetonitrile/water. For experiments performed with ethidium bromide and methylene blue, the oligonucleotide solution was mixed with an equal volume of the additive solution prior to spotting on the sample plate. For those experiments using peptides as additives, a microliter of a peptide stock solution was combined with a microliter of the stock double-stranded oligonucleotide solution. When incubated, the peptide/oligonucleotide solution was heated to $37{ }^{\circ} \mathrm{C}$ for $15 \mathrm{~min}$. A microliter of the resulting solution was spotted onto the MALDI target with a microliter of the matrix solution.

Linear MALDI mass spectra were recorded on a PerSeptive Biosystems (Framingham, MA) Voyager delayed-extraction time-of-flight (TOF) linear mass spectrometer equipped with a nitrogen laser $(337 \mathrm{~nm}, 3 \mathrm{~ns}$ pulse). For the negative ion MALDI spectra reported here, the accelerating voltage was $-15 \mathrm{kV}$, the delay time, selected for optimum resolution, was 700 ns [3], the grid voltage was $94.5 \%$ of the accelerating voltage, 


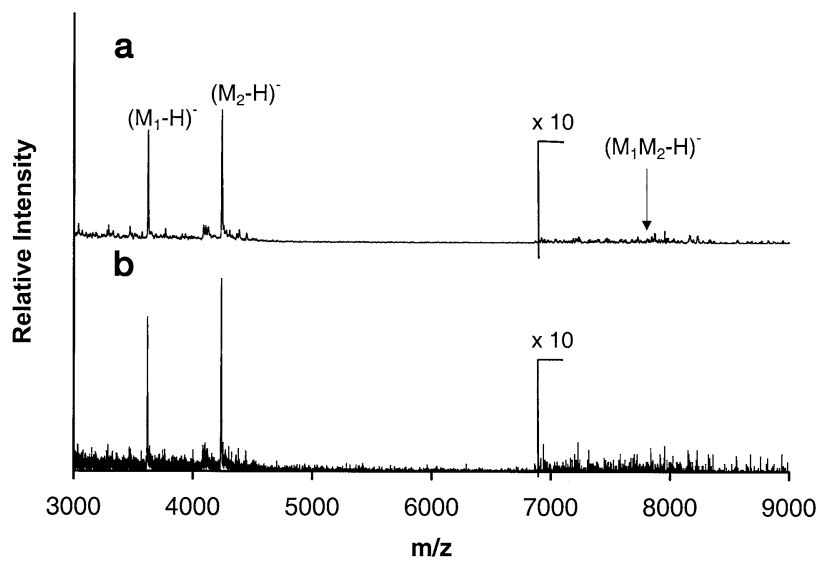

Figure 1. The negative-ion UV MALDI mass spectra of duplex 1 using (a) ATT and (b) HPA as matrices. Duplex 1 consists of two strands with molecular weights of $3646 \mathrm{~g} / \mathrm{mol}$ and $4254 \mathrm{~g} / \mathrm{mol}$. The strand with the lower molecular weight is referred to as $M_{1}$ while the strand with the larger molecular weight is referred to as $\mathrm{M}_{2}$. No duplex ions are detected (position indicated by arrow) giving a DSRR of 0 .

and the magnitude of the guide wire voltage was $0.20 \%$ of the accelerating voltage. Typically, transients from 50 laser shots were averaged for each spectrum.

Melting temperature studies and UV analyses were performed using an ATI Unicam (Cambridge, UK) UV2 spectrophotometer. Oligonucleotides have strong UV absorption maxima at $259 \mathrm{~nm}$. This absorption arises almost entirely from the complex electronic transitions in the purine and pyrimidine components [23]. In double-stranded oligonucleotides, the base-base stacking results in a decrease in molar absorptivity compared to that for the two single-stranded components. This is known as the hyperchromic effect. For melting studies, UV spectra were taken using the oligonucleotide solutions at room temperature. The analyte solutions were then heated to $90{ }^{\circ} \mathrm{C}$ in a sand bath for $10 \mathrm{~min}$ in order to denature the double-stranded oligonucleotides. If the duplex existed at room temperature, the absorbance should increase by $10 \%$ due to the unstacking of the aromatic bases upon denaturation. To insure renaturation of the duplex, the absorbance can again be recorded after the solution returns to room temperature. All spectra were acquired from 200 to $400 \mathrm{~nm}$ with a scan speed of $120 \mathrm{~nm} / \mathrm{min}$, a data interval of $0.5 \mathrm{~nm}$, and a $2.0 \mathrm{~nm}$ bandwidth. Quartz cuvettes with a $1 \mathrm{~cm}$ path length were used, with water in the reference cuvette.

\section{Results and Discussion}

When analyzing duplex 1 using ATT and HPA as matrices, only peaks representing the two single strands are detected, Figure $1 \mathrm{a}$ and $\mathrm{b}$ respectively. While these matrices have previously shown promise for the detection of non-covalent complexes in UV MALDI MS [12, 13], the duplex is not preserved in these experiments. This may be related to size differences between the two experiments. Similar results are achieved with duplex 2; no intact duplex peaks are detected even though these duplexes have melting points which indicate that they are stable at room temperature. When analyzing duplex 3 , a small peak representing the duplex with a loss of a guanine is seen. No peak is detected representing the intact $\left(\mathrm{M}_{1} \mathrm{M}_{2}-\mathrm{H}\right)^{-}$species. All the experiments were completed with the same level of double-stranded oligonucleotide. We have examined the correlation between the amount of duplex deposited on the target and the double-stranded region of the spectrum and have found no influence, when working in a normal range for MALDI samples (1-100 pmol of analyte).

Due to the negatively charged phosphate backbone, negative-ion mode is often used to study oligonucleotides in MALDI MS and will be used in this discussion. When analyzing the annealed double-stranded species, $\mathrm{M}_{1} \mathrm{M}_{2}$, in negative-ion mode, ions representing the single strands, $\left(\mathrm{M}_{1}-\mathrm{H}\right)^{-}$and $\left(\mathrm{M}_{2}-\mathrm{H}\right)^{-}$, dominate the spectrum. Negative-ion spectra exhibit better resolution and a higher signal-to-noise ratio. In order to measure the success of an experiment, several criteria will be used when an additive allows for the detection of duplex ions. The strand with the lower molecular weight will be denoted as $\mathrm{M}_{1}$ while the larger complementary strand will be denoted as $\mathrm{M}_{2}$. The ratio of the intensity (I) of the $\left(\mathrm{M}_{1}-\mathrm{H}\right)^{-}$peak to the intensity of the $\left(\mathrm{M}_{2}-\mathrm{H}\right)^{-}$peak is of interest. In previous experiments, our laboratory has analyzed double-stranded oligonucleotides in the UV-MALDI experiment where only ions from one of the strands are formed. This result is unexpected since the complementary strands are present in equal amounts and are of comparable molecular weights. This effect appears to depend on both the sequence of the oligonucleotides and the matrix used in the experiment. The ratio of the intensity of the $\left(\mathrm{M}_{1}-\right.$ $\mathrm{H})^{-}$peak to the intensity of the $\left(\mathrm{M}_{2}-\mathrm{H}\right)^{-}$peak is referred to as the single strand distribution (SSD), eq 1.

$$
\mathrm{SSD}=\frac{\mathrm{I}\left(\mathrm{M}_{1}\right)}{\mathrm{I}\left(\mathrm{M}_{2}\right)}
$$

Note that the nomenclature used in eq 1 is not, technically, correct. We chose to write $\mathrm{I}\left(\mathrm{M}_{1}\right)$ rather than $\mathrm{I}\left(\mathrm{M}_{1}-\mathrm{H}\right)^{-}$, for example, to allow the quantifier to be used in either negative or positive ion experiments.

To the extent that the single strand ions may evolve from the duplex, we would like to monitor how the SSD value correlates with other variables in the experiment, and to determine if the SSD correlates with single strand composition. If, for example, the single strand ions are fragments of the singly charged gas phase duplex, then one may expect that the decision of which strand retains the charge would be determined by the base composition of the individual strands. On the other hand, if duplex dissociation occurs early in the experiment, one may anticipate an SSD of 1.0. For the spectra shown in Figure 1a and b, the SSD values are 


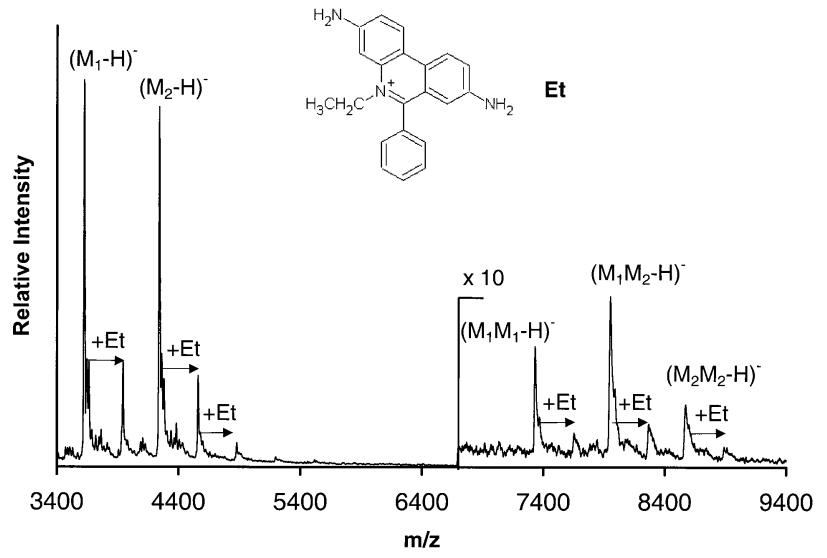

Figure 2. The negative-ion MALDI mass spectrum of duplex 1. HPA was used as the matrix with ethidium (Et) bromide as an additive. Ethidium adducts of both the single and doublestranded oligonucleotides are observed. The SSD is 1.0, DSRR is 0.05 , and the WCSF is 0.5 . The additive duplex ratio is $40: 1$.

both 0.9. That is, D/I of the larger strand is slightly favored.

The goal of this project is to optimize the ratio of the intensity of the double strand peak to the intensities of the single strand peaks. This double strand retention ratio (DSRR) is defined in eq 2 .

$$
\operatorname{DSRR}=\frac{\mathrm{I}\left(\mathrm{M}_{1} \mathrm{M}_{2}\right)}{\mathrm{I}\left(\mathrm{M}_{1}\right)+\mathrm{I}\left(\mathrm{M}_{1} \mathrm{M}_{2}\right)+\mathrm{I}\left(\mathrm{M}_{2}\right)}
$$

While the SSD values were 0.9 in Figure $1 \mathrm{a}$ and $b$, the DSRR was zero for both experiments. The most intense analyte-related peaks in the spectrum represent the $\left(\mathrm{M}_{1}-\mathrm{H}\right)^{-}$and $\left(\mathrm{M}_{2}-\mathrm{H}\right)^{-}$ions. The duplex must have dissociated before the acceleration and detection of the ions. The presence of stabilizing additives in the target may hold the complex together. Several experiments were performed using stabilizing additives in order to study their effect on the SSD and DSRR values for each spectrum.

As mentioned previously, ethidium bromide has been found to stabilize duplexes in solution. When used in the MALDI experiment, equal volumes of duplex 1 and ethidium bromide solutions were combined. One microliter of the oligonucleotide:ethidium solution was spotted on the MALDI target with one microliter of the HPA matrix solution. The molar amounts of components of the target are matrix:additive:oligo, 2000:40:1. This is typical since effective additives for MALDI MS are usually present at molar amounts greater than the analyte. The resulting spectrum is shown in Figure 2, with the structure of the ethidium ion. The ethidium cation binds to both the single and double-stranded species of duplex 1 . That is, in addition to analyte ions, $(\mathrm{A}-\mathrm{H})^{-}$, there are also $(\mathrm{A}-2 \mathrm{H}+\mathrm{Et})^{-}$ions observed. With the appearance of the dimer peaks, the DSRR increases from 0 (in Figure $1 b$ ) to 0.05 .

While the DSRR increases with the addition of ethidium bromide, the goal of this work is to retain the maximum extent of Watson-Crick base pairing when detecting a double-stranded species. If the $\mathrm{M}_{1} \mathrm{M}_{2} \mathrm{du}$ plex completely dissociates during crystal formation, some fraction of $M_{1}$ and $M_{2}$ may recombine in a non-Watson-Crick pairing. The single-stranded species, $\mathrm{M}_{1}$ and $\mathrm{M}_{2}$, can, and clearly do, form three different dimers: $M_{1} M_{1}, M_{1} M_{2}$, and $M_{2} M_{2}$. In order to quantify the amount of Watson-Crick pairing involved, we will calculate a Watson-Crick selectivity factor (WCSF), eq 3.

$$
\text { WCSF }=\frac{I\left(M_{1} M_{2}\right)}{I\left(M_{1} M_{1}\right)+I\left(M_{1} M_{2}\right)+I\left(M_{2} M_{2}\right)}
$$

If the experiment begins with pure duplex and complete dissociation occurs, during crystal growth, then there will be equimolar amounts of $\mathrm{M}_{1}$ and $\mathrm{M}_{2}$ available in the solution on the sample plate. If random dimer formation occurs, a WCSF value of 0.5 would be expected. This suggests that the distribution of duplex peaks was dictated by random condensation events. Even in peptide analysis, non-specific dimerization has been observed [27]. As the value of the WCSF approaches 1, Watson-Crick base pairing is preserved and, presumably, the non-covalent forces were maintained throughout the MALDI experiment. When changing the variables in the MALDI experiment, the value of the WCSF provides information about the nature of the species detected and gives direction to subsequent experiments.

In Figure 2, while there are peaks representing the $\left(\mathrm{M}_{1} \mathrm{M}_{2}-\mathrm{H}\right)^{-}$species, there are also peaks representing the $\left(\mathrm{M}_{1} \mathrm{M}_{1}-\mathrm{H}\right)^{-}$and $\left(\mathrm{M}_{2} \mathrm{M}_{2}-\mathrm{H}\right)^{-}$species. While the DSRR increased from 0 to 0.05 , the WCSF is 0.5 , suggesting random dimerization. If random dimerization occurs, the duplex may completely dissociate and the single strands are likely dimerizing with no sequence specificity as the crystals are growing. Thus, while ethidium addition does result in "dimer ions", we conclude that this additive does not stabilize the initial duplex throughout the UV-MALDI experiment.

Other compounds known to bind to double-stranded DNA [23] were evaluated as matrix additives as well including chromomycin, distamycin, berenil, daunomycin, and methylene blue. Of these compounds, distamycin and berenil were found to bind to the singlestranded oligonucleotides in the UV-MALDI experiment. There were, however, no duplex or dimer peaks detected, giving a DSRR of zero for both additives. The SSD was 0.7 for the berenil experiment and 1.0 for the distamycin experiment. Chromomycin and daunomycin had no apparent effects on the experiment.

Methylene blue was also found to bind to the singlestranded oligonucleotide. The experimental procedure used for the ethidium bromide experiments was followed using methylene blue as the additive. The solution was then spotted with ATT as the matrix. The resulting spectrum is shown in Figure 3, with the 


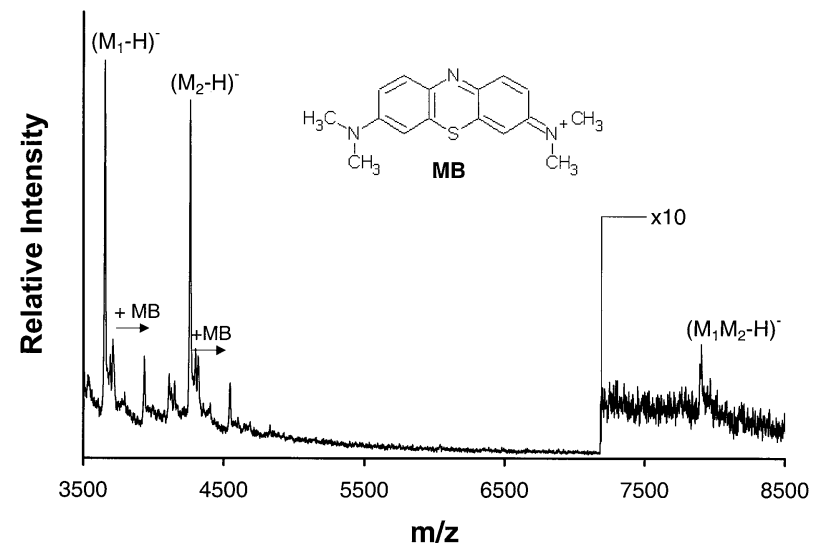

Figure 3. The negative-ion MALDI mass spectrum of duplex 1 with methylene blue as an additive. ATT was used as the matrix. The SSD is 0.9 , the DSRR is 0.03 , and the WCSF is 1.0. The additive duplex ratio is 40:1.

structure of the methylene blue cation. While methylene blue (MB) is observed bound to the single-stranded species, its presence appears to stabilize the duplex as well, Figure 3. In this experiment, the DSRR value was 0.03 , the SSD was 0.9 , and the WCSF was 1.0. With a WCSF of 1.0, it appears that a portion of the duplex was maintained throughout the entire UV-MALDI experiment, due to the presence of this additive, although the duplex represents only a small percentage of the initial complex.

In addition to intercalating and groove-binding molecules, spermine was also found to be an effective additive. When spermine is used with ATT, there are significant changes in the double-stranded region of the spectrum. Figure 4 a shows the spectrum of duplex 3 when ATT and spermine are used as a matrix and Figure $4 \mathrm{~b}$ shows a spectrum of the same duplex with ATT as the matrix. Without spermine, a peak representing a duplex fragment, due to the loss of a guanine, is

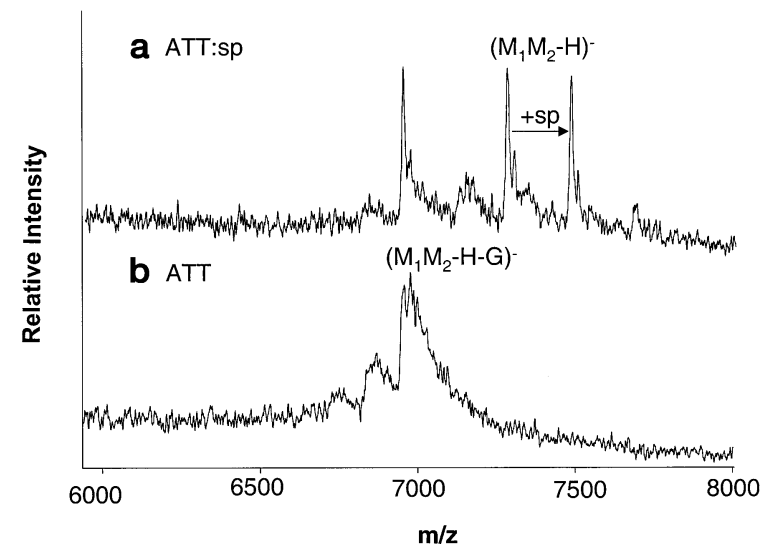

Figure 4. Portions of the negative-ion MALDI mass spectra of duplex 2 using (a) ATT/sp and (b) ATT as the matrix. Note the intact duplex ion is formed when spermine is present while only a duplex ion fragment is formed with ATT alone. In the ATT/sp experiment, the DSRR is 0.10 and the WCSF is 1.0. The SSD is 1.0 for both experiments. The additive:duplex ratio is 500:1. seen. When spermine is present, two additional peaks are seen. The intact, deprotonated duplex, $\left(\mathrm{M}_{1} \mathrm{M}_{2}-\right.$ $\mathrm{H})^{-}$is detected as well as its spermine (sp) adduct, $\left(\mathrm{M}_{1} \mathrm{M}_{2}-\mathrm{H}+\mathrm{sp}\right)^{-}$. Compared to Figure 1a, the DSRR increases from zero to 0.1 . The SSD values are 1.0 for both experiments. The WCSF value for this spectrum is 1 , with only high mass peaks relating to the $M_{1} M_{2}$ species being detected.

While spermine may contribute to duplex stability in the crystal growth event, the detection of $\left(\mathrm{M}_{1} \mathrm{M}_{2}-\mathrm{H}+\right.$ $\mathrm{sp})^{-}$suggests that a spermine complex was formed and remained intact through the D/I event. The effect is duplex-dependent. Spermine stabilizes duplex 3, but when ATT:sp is used in the analysis of duplex 1 or 2, ions representing the intact duplex are not exclusively detected; peaks representing $\left(\mathrm{M}_{1} \mathrm{M}_{1}-\mathrm{H}\right)^{-}$and $\left(\mathrm{M}_{2} \mathrm{M}_{2}-\mathrm{H}\right)^{-}$are detected as well.

In Figure $4 \mathrm{a}$, while most of the duplex dissociated, some duplex ions were detected in the MALDI analysis. The addition of spermine to the matrix has, at some point in the experiment, allowed for the detection of what appears to be authentic double-stranded DNA ions with Watson-Crick base-pairing preserved. The improvement in resolution when spermine is added may suggest that spermine-duplex adducts are trapped in the crystals. This may lower the average number of negative charges on trapped oligonucleotides, simplifying desorption kinetics.

While spermine stabilizes the duplex during the MALDI experiment, it also acts to effectively displace alkali ions, decreasing the abundance of sodium and potassium adducts [2]. Spermine may serve an important role in the eventual development of a mixed matrix for duplex analysis. Addition of spermine is preferable to sample purification. In analyzing other biopolymers by MS, it is certainly common to purify by, for example, ion exchange if a sample has a high salt content. However, when the analyte is a DNA duplex, this may not be a logical procedure to use. Ions such as $\mathrm{Na}^{+}$and $\mathrm{K}^{+}$stabilize duplexes; melting points are lowered when salts are removed [28]. Thus, desalting may well decrease the success of a duplex analysis by UV-MALDI MS. Spermine allows for some salt to be present, and displaces alkali ions as the target is formed.

In the evaluation of spermine as an additive, Figure 4 , the double-stranded oligonucleotide is detected in both experiments. In Figure $4 b$, the presence of salts interferes with the analysis. The duplex oligonucleotide may form many alkali ion adducts leading to decreased resolution and signal-to-noise ratio. The presence of spermine in Figure 4a leads to an improvement in the resolution. The presence of spermine in the matrix solution may also improve the results of experiments using other additives such as methylene blue. If the experiment in Figure 3 is repeated with a matrix solution containing spermine, the resolution in the duplex region is improved, the DSRR value increases, and a methylene blue adduct is detected in the duplex region, Figure 5. The DSRR increases significantly from 0.03 in 


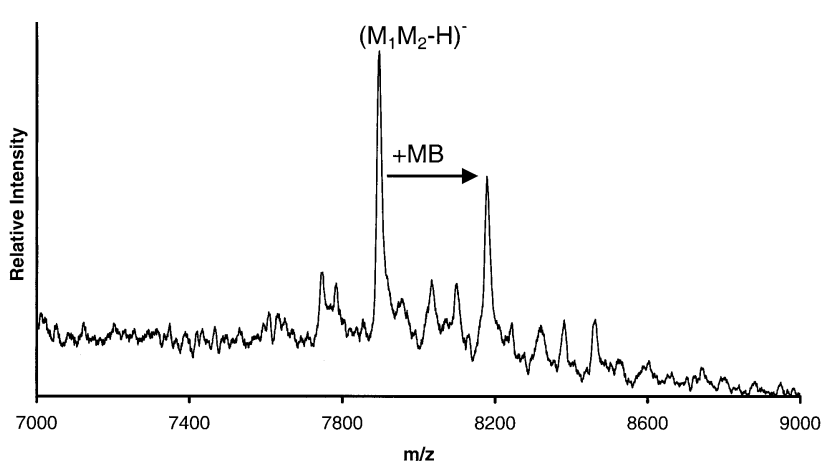

Figure 5. A portion of the negative-ion MALDI mass spectrum of duplex 1 with methylene blue (MB) as an additive. ATT:sp was used as the matrix. The DSRR is 0.05 and the WCSF is 1.0. The spermine:MB:duplex ratio is 500:40:1.

Figure 3 to 0.07 in Figure 5. The WCSF is 1.0 and the SSD is 0.9 .

There have been many cases in UV-MALDI MS where a complex is observed, possibly as an analyte with a matrix molecule or analyte with an additive. It may be significant that, when the additive MB appears to allow intact duplex detection, there is direct evidence in the spectrum for existence of the $\mathrm{M}_{1} \mathrm{M}_{2} \cdot \mathrm{MB}$ complex. This suggests that MB forms a strong complex with the duplex that is more stable than the duplex alone, and that it is involved in duplex preservation in the target preparation step.

If some small molecules that are known to interact with DNA can stabilize the duplex in the MALDI experiment, larger molecules such as proteins and peptides may also have a stabilizing effect. In an experiment designed in our laboratory to explore the differences in resolution and sensitivity between a peptide and an oligonucleotide in UV-MALDI-MS, a solution was prepared that contained equal amounts of a peptide and oligonucleotide of similar molecular weights. In the resulting spectra, peaks were detected for the peptide, $(\mathrm{P}+\mathrm{H})^{+}$, the oligonucleotide, $(\mathrm{O}+\mathrm{H})^{+}$, and a peptide-oligonucleotide complex, $(\mathrm{P}+\mathrm{O}+\mathrm{H})^{+}$. Similar results have been reported previously [29]. In order to examine the influence of peptides on doublestranded oligonucleotides, several peptides were combined with duplex oligonucleotides and analyzed by UV MALDI-MS. The peptides were used at a level of 1 pmol or less. An additive that is most effective at a level similar to that of the analyte, rather than that of the matrix, will be referred to as a microadditive (with the hope that there will not be a future need to separately define nanoadditives, picoadditives, etc).

Duplex 1 was used for the experiments designed to evaluate peptides as microadditives with ATT:spermine as the matrix, Figure 6a. The SSD is 0.7 and the DSRR is 0.006 . While the intact duplex, $\left(\mathrm{M}_{1} \mathrm{M}_{2}-\mathrm{H}\right)^{-}$is detected in this experiment, the peak representing the $\left(\mathrm{M}_{2} \mathrm{M}_{2}-\mathrm{H}\right)^{-}$species is surprisingly intense, giving the spectrum a WCSF of 0.3 .

When only 1 pmol of $\beta$-MSH was added to the target

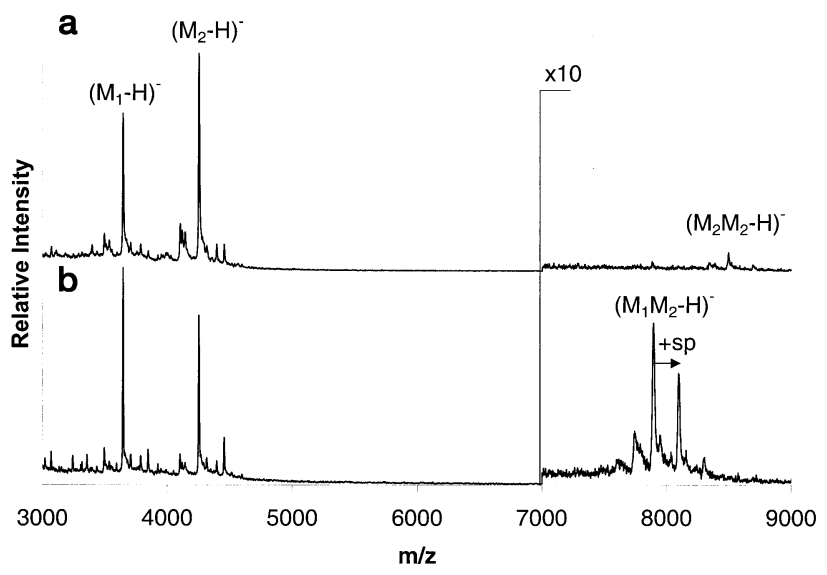

Figure 6. Negative-ion MALDI mass spectra of duplex 1 with (a) no peptide present and (b) with $\beta$-MSH present. ATT:sp was used as the matrix for both experiments. 25 pmol of duplex and 1 pmol of $\beta$-MSH were deposited onto the MALDI target. For (a), the SSD is 0.7 , the DSRR is 0.006 , and the WCSF is 0.3 . For $(\mathbf{b})$, the SSD is 1.1 , the DSRR is 0.06 , and the WCSF is 1.0. The peptide:duplex ratio is $1: 25$.

(which contained 25 pmol of duplex) used to obtain Figure $6 \mathrm{a}$, the spectrum shown in Figure $6 \mathrm{~b}$ is obtained. The SSD changes dramatically to 1.1, the DSRR is 0.06, and the WCSF is 1.0. There are no peaks representing either $\left(\mathrm{M}_{1} \mathrm{M}_{1}-\mathrm{H}\right)^{-}$or $\left(\mathrm{M}_{2} \mathrm{M}_{2}-\mathrm{H}\right)^{-}$. In this experiment, $\beta$-MSH stabilized, to some extent, doublestranded DNA ions in UV MALDI MS, when present at a level of one picomole on the MALDI target. Other peptides evaluated are listed in Table 2. All peptides were used as additives by adding $1 \mathrm{pmol}$ of peptide to $25 \mathrm{pmol}$ of duplex 1 and analyzed using ATT:sp as the matrix. The DSRR and SSD values are listed in the table. All WCSF values are 1.

The peptides evaluated as microadditives can be grouped based both on their size and amino acid content. It is possible that the most successful experiments would have involved peptides that have a size similar to that of the oligonucleotide. From spermine experiments performed previously [2, 3], we have learned that additives capable of multiple interactions with the oligonucleotides are important. For this reason, the number of basic residues in the peptide may be important, with multiple basic residues increasing the number of electrostatic interactions possible. Previously it was determined that peptides containing basic residues increase the melting temperature of duplex oligonucleotides in solution [30]. Protonated backbone nitrogens may aid in stabilization as well.

When examining the data in Table 2, attempts were made to correlate the experimental results with the structural features of the peptides. The two peptides used with the most notable success were dynorphin A and $\beta$-MSH. Both of these larger peptides contain basic residues with three arginines and two lysines out of the 17 amino acids in dynorphin $\mathrm{A}$ and six basic residues out of the 22 amino acids residues in $\beta$-MSH including three lysines, two arginines, and one histidine. Dynor- 
Table 2. Peptide additives and their influence on UV MALDI spectra of duplex 1

\begin{tabular}{|c|c|c|c|c|c|c|c|}
\hline Peptide & Peptide sequence & SSD & WCSF & DSRR & $\% \operatorname{Arg}$ & $\%$ Lys & MW \\
\hline Dynorphin A & YGGFLRRIRPKLKWDNO & 1.1 & 1.0 & 0.1 & 17.65 & 11.76 & 2147.50 \\
\hline$\beta$-MSH & AEKKDEGPYRMEHFRWGSPPKD & 1.1 & 1.0 & 0.1 & 9.09 & 13.64 & 2660.90 \\
\hline Katacalcin & DMSSDLERDHRPHVSMPQNAN & 1.1 & 1.0 & 0.0 & 9.52 & 0.00 & 2436.60 \\
\hline Kemptide & LRRASVA & 0.9 & 1.0 & 0.0 & 28.57 & 0.00 & 771.90 \\
\hline FBI peptide & HHLGGAKQAGDV & 1.0 & 1.0 & 0.0 & 0.00 & 8.33 & 1189.30 \\
\hline Trilysine & KKK & 1.0 & 1.0 & 0.0 & 0.00 & 100.00 & 402.50 \\
\hline $\mathrm{N}$ - $\varepsilon$-acetyl-Lys & $\mathrm{K}$ & 1.0 & 1.0 & 0.0 & 0.00 & 100.00 & 188.23 \\
\hline Leucine enkephalin & YGGFL & 1.0 & 1.0 & 0.0 & 0.00 & 0.00 & 555.60 \\
\hline Boc-MNF amide & BMNF & 0.8 & 1.0 & 0.0 & 0.00 & 0.00 & 510.60 \\
\hline Hexaalanine & AAAAAA & 1.0 & 1.0 & 0.0 & 0.00 & 0.00 & 444.50 \\
\hline Trialanine methyl ester & AAA & 0.9 & 1.0 & 0.0 & 0.00 & 0.00 & 305.30 \\
\hline Triserine & SSS & 1.1 & 1.0 & 0.0 & 0.00 & 0.00 & 279.20 \\
\hline
\end{tabular}

phin A and $\beta$-MSH presumably interact with the double-stranded oligonucleotides at multiple sites. The backbone nitrogens may be important in the stabilization of the duplex. A positively-charged backbone in a peptide may behave similarly to spermine in solution. The side chains of the peptide may also play an important role. The amino acids, tyrosine, phenylalanine, and tryptophan, contain aromatic side chains. These side chains may partially intercalate between the nitrogencontaining bases of the oligonucleotide, stabilizing the duplex. Increases in the melting temperature of duplex DNA have been reported when in the presence of peptides containing phenylalanine residues [31]. Basic side chains may also play an important role as discussed previously.

Since peptides containing only alanine residues were not as successful as additives, it appears that the backbone nitrogens are not effective in stabilizing the duplex. Small peptides containing basic residues such as Lys-Lys-Lys were found to increase the DSRR value although these smaller peptides were less effective than Dynorphin A and $\beta$-MSH. Similarly, peptides containing aromatic side chains were also capable of increasing the DSRR values for the experiments.

Since the best results are seen with the peptides Dynorphin A and $\beta$-MSH, which contain both aromatic and basic amino acids, it is not perhaps the size of the peptide that is of primary importance, but the spacing between basic amino acids. When basic amino acids in a larger peptide are separated by other residues, the separation between those basic residues may be similar to the spatial separation between the phosphates of the oligonucleotide. This may lead to greater stabilization of the duplex. Clearly, more work is required to find the optimal peptide, but these experiments establish structural aspects of the additives that are important and show that peptides can, at relatively low levels, stabilize to some extent duplexes in the UV MALDI experiment.

In order to maximize the interaction between the peptides and the double-stranded oligonucleotides, several conditions were varied in the experiments. At first the peptide and oligonucleotide were combined on the MALDI plate before the addition of the matrix. To insure thorough mixing, peptide and oligonucleotide solutions were also premixed before deposition on the target. There was no difference between spectra acquired using on target mixing or premixed solutions. A solution of the peptide and oligonucleotide was also pre-mixed and incubated at both room temperature and at $37{ }^{\circ} \mathrm{C}$ prior to MALDI analysis. When incubated at either temperature, samples again yielded spectra similar to the unincubated samples.

For the data shown in Table 2, one picomole of each peptide was used in the formation of the MALDI target. While 1 pmol was optimal for most peptides, improvements were seen with as little as $50 \mathrm{fmol}$ of peptides present in the target. FBI peptide, katacalcin, and $\beta$-MSH all yielded duplex spectra with improved DSRR values with less than 1 pmol of peptide present in the target. While addition of these peptides to the MALDI target improved the DSRR, the $\beta$-MSH peptide gave the best results. Figure 7 shows the negative-ion MALDI mass spectrum of a target containing $100 \mathrm{fmol}$ of $\beta$-MSH and 25 pmol of double-stranded oligonucleotide, using ATT:sp as the matrix. The SSD was 0.94, the DSRR was 0.09 and the WCSF was 1.00. The presence of

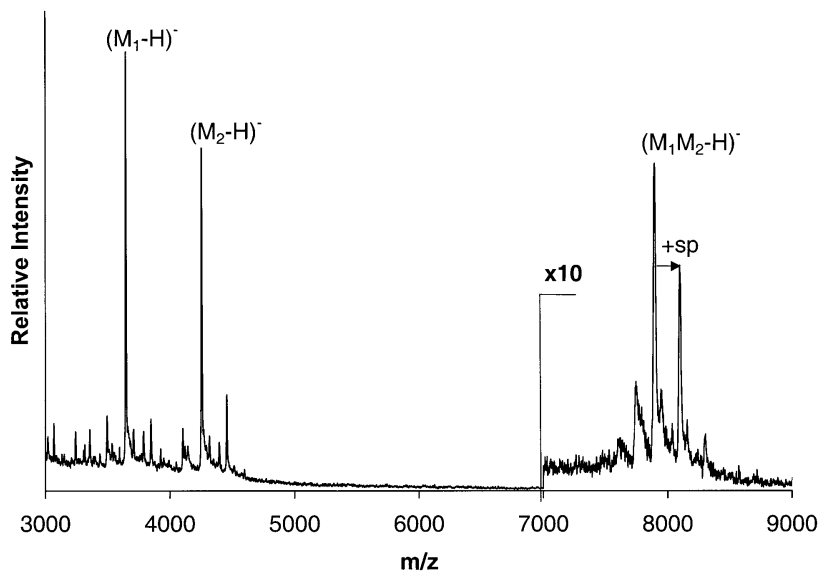

Figure 7. Negative-ion MALDI mass spectrum of duplex 1 with $100 \mathrm{fmol}$ of $\beta$-MSH and ATT:sp as the matrix. The SSD is 0.94, the DSRR is 0.09 , and the WCSF is 1.00 . The peptide:duplex ratio is $1: 250$. 
the peptide still influences the resulting mass spectrum even when the relative concentration of the oligonucleotide:peptide is 250:1.

All of the experiments reported here were performed on a single "batch" of a given duplex. We have purchased synthetic, annealed duplexes from both an oncampus facility and from commercial suppliers. In the case of the Michigan State University Facility, oligonucleotides are usually synthesized as primers and we are one of a few groups on campus that request synthesized strands and their annealed duplexes. We have found that some sources occasionally provide a duplex that is more stable than the same duplex in another batch. Salts and buffers that are commonly provided in such samples, while not specified, could alter resulting MALDI spectra. All of the spectra presented here have been duplicated on multiple dates from the same sample, and from other batches of the same sample, to confirm that no result was influenced by an impurity or contaminant in a single batch. From multiple experiments on identical samples, we believe that the quantities reported here are reflections of the relative influence of the additives discussed. Spectra were obtained from several locations across the target. Those shown represent an average for the target. In identical experiments, the WCSF and SSD quantifiers are reproducible to $10 \%$. For example, in Figure 7, the WCSF value was $1.0 \pm 0.1$ and the SSD has a value of $0.94 \pm 0.90$. This was not the case for the DSRR values. For the experiments shown here, the DSRR values reported ranged from 0 to 0.1 . The experiments that yielded DSRR values of zero did so consistently. When duplex peaks were detected in an experiment, the DSRR values were reproducible to $25 \%$. For the additives shown, typically $5-10 \%$ of the duplex molecules were stabilized in the experiment, and can be viewed as having similar stabilizing capabilities.

\section{Conclusion}

Methylene blue, spermine, and $\beta$-MSH as additives allow for the detection of small intact DNA duplexes. Each of these compounds must be capable of stabilizing double-stranded DNA in at least the crystal growth process, and possibly in the desorption/ionization process as well. While these additives all increase the DSRR values, they may participate in a different way throughout the MALDI process. For example, spermine aids in the crystallization of the duplex, and complexes with the oligonucleotide during crystal growth. During the desorption/ionization of the oligonucleotide, the spermine may dissociate from the duplex, resulting in the detection of the intact duplex, $\left(\mathrm{M}_{1} \mathrm{M}_{2}-\mathrm{H}\right)^{-}$. The spermine may also stabilize the duplex through the desorption/ionization process with the $\left(\mathrm{M}_{1} \mathrm{M}_{2}+\mathrm{sp}-\right.$ $\mathrm{H})^{-}$species being detected. In order for the spermine to bind to the DNA and stabilize it, the spermine must be present at a concentration equal to, or greater than, the oligonucleotide concentration. In contrast, peptides are capable of stabilizing the duplex oligonucleotides even when present at concentrations less than that of the oligonucleotide. The peptides may stabilize the duplex through another mechanism. If the peptide can stabilize the duplex whether it is present at a level of 1 pmol or $50 \mathrm{fmol}$ (always present at a level less than the $25 \mathrm{pmol}$ of duplex used for each experiment presented here), each peptide may be capable of interacting with more than one duplex throughout the experiment. Since the peptide is needed in only a catalytic amount, it may be acting as a chaperone for the double-stranded oligonucleotides. The peptide may bind and stabilize the duplex during the crystal growth step. After depositing the duplex on the growing crystal surface, the peptide may dissociate from the duplex, allowing it to interact with another duplex still in solution.

Alternatively, the equilibrium constant for a duplexspermine complex may be much smaller than that for a duplex-peptide complex. Thus, to achieve the same effect, a greater concentration of spermine is needed, although the mechanism is the same. Even speculating on this possibility is difficult because it is not known how many additive molecules interact with a single duplex. In order to fully understand this process, the stoichiometry of the critical step must be known.

Since peptide-duplex complexes are not detected in these MALDI experiments, a chaperone mechanism is more likely. While it may be different from the mechanism for spermine, the peptide-oligonucleotide interaction, in some ways, parallels the behavior of spermine. When crystals of oligonucleotides are required for X-ray crystallographic studies, spermine is frequently added to facilitate crystal growth. However, spermine is usually not found in the crystals that are formed. Multiply protonated, positively-charged spermines presumably interact with the oligonucleotides, but the spermine molecules are efficiently eliminated as the crystals grow. In a similar way, peptides such as $\beta$-MSH may bind with duplexes only until they become incorporated into the matrix crystal, and become released at that time.

Why do DNA duplexes dissociate in the UV MALDI experiment? Results presented here suggest that extensive, if not complete, dissociation occurs in the first step during growth of the crystal target. We have investigated the possibility that the duplexes dissociate in the initial solution due to the organic solvents and excess matrix molecules, but these do not appear to be sufficient for inducing dissociation. One possibility is the crystals themselves. When a duplex lands on a growing crystal in the initial solution, multiple possibilities follow. It may desorb from the surface in duplex form, it may incorporate into the crystal in duplex form, or it may dissociate, leaving one strand bound to the surface. If the matrix crystals are responsible for dissociation of non-covalent complexes that are analyte in a MALDI experiment, methods for more rapid crystal growth could result in more efficient trapping of such complexes for subsequent analysis. 


\section{References}

1. Karas, M.; Bachmann, D.; Bahr, U.; Hillenkamp, F. MatrixAssisted Ultraviolet-Laser Desorption of Non-Volatile Compounds. Int. J. Mass Spectrom. 1987, 78, 53-68.

2. Asara, J. M.; Allison, J. Enhanced Detection of Oligonucleotides in UV MALDI MS Using Tetraamine Spermine as a Matrix Additive. Anal. Chem. 1999, 71, 2866-2870.

3. Distler, A. M.; Allison, J. 5-Methoxysalicylic Acid and Spermine: A New Matrix for the Matrix-Assisted Laser Desorption/ Ionization Mass Spectrometry Analysis of Oligonucleotides. J. Am. Soc. Mass Spectrom. 2001, 12, 456-462.

4. Distler, A. M.; Allison, J. Improved MALDI-MS Analysis of Oligonucleotides through the Use of Fucose as a Matrix Additive. Anal. Chem. 2001, 73, 5000-5003.

5. Shahgholi, M.; Garcia, B. A.; Chiu, N. H. L.; Heaney, P. J.; Tang, K. Sugar Additives for MALDI Matrices Improve Signal Allowing the Smallest Nucleotide Change (A:T) in a DNA Sequence to be Resolved. Nucleic Acids Res. 2001, 29, e91/1e91/10.

6. Koomen, J. M.; Russell, W. K.; Hettick, J. M.; Russell, D. H. Improvement of Resolution, Mass Accuracy, and Reproducibility in Reflected Mode DE-MALDI-TOF Analysis of DNA Using Fast Evaporation-Overlayer Sample Preparations. Anal. Chem. 2000, 72, 3860-3866.

7. Zhang, L. K.; Gross, M. L. Matrix-Assisted Laser Desorption/ Ioniation Mass Spectrometry Methods for Oligodeoxynucleotides: Improvements in Matrix, Detection Limits, Quantification, and Sequencing. J. Am. Soc. Mass Spectrom. 2000, 11, 854-865.

8. Pieles, U.; Zürcher, W.; Schär, M.; Möser, H. E. MatrixAssisted Laser Desorption Ionization Time-of-Flight Mass Spectrometry: A Powerful Tool for the Mass and Sequence Analysis of Natural and Modified Oligonucleotides. Nucl. Acids Res. 1993, 21, 3191-3196.

9. Yi, Y. C. L.; Cheng, S.; Chan, T. W. D. Evaluation of Ammonium Salts as Co-matrices for Matrix-Assisted Laser Desorption/Ionization Mass Spectrometry of Oligonucleotides. Rapid. Commun. Mass Spectrom. 1998, 12, 993-998.

10. Cheng, S. W.; Chan, T. W. D. Use of Ammonium Halides as Co-matrices for Matrix-Assisted Laser Desorption/Ionization Studies of Oligonucleotides. Rapid Commun. Mass Spectrom. 1996, 10, 907-910.

11. Vandell, V. E.; Limbach, P. A. Polyamine Co-matrices for Matrix-Assisted Laser Desorption/Ionization Mass Spectrometry of Oligonucleotides. Rapid Commun. Mass Spectrom. 1999, 13, 2014-2021.

12. Lecchi, P.; Panell, L. K. The Detection of Intact DoubleStranded DNA by MALDI. J. Am. Soc. Mass Spectrom. 1995, 6, 972-975.

13. Kirpekar, F.; Berkenkamp, S.; Hillenkamp, F. Detection of Double-Stranded DNA by IR- and UV-MALDI Mass Spectrometry. Anal. Chem. 1999, 71, 2334-2339.

14. Little, D. P.; Jacob, A.; Becker, T.; Braun, A.; Darnhofer-Demar, B.; Jurinke, C.; van den Boom, D.; Koster, H. Direct Detection of Synthetic and Biologically Generated Double-Stranded
DNA by MALDI-TOF MS. Int. J. Mass Spectrom. Ion Processes 1997, 169/170, 323-330.

15. Tang, K.; Taranenko, N. I.; Allman, S. L.; Chen, C. H.; Chang, L. Y.; Jacobson, K. B. Picolinic-Acid as a Matrix for Laser Mass-Spectrometry of Nucleic-Acids and Proteins. Rapid Commun. Mass Spectrom. 1994, 8, 673-677.

16. Shui, X.; McFail-Isom, L.; Hu, G. G.; William, L. D. The B-DNA Dodecamer at High Resolution Reveals a Spine of Water on Sodium. Biochemistry 1998, 37, 8341-8355.

17. Halle, B.; Denisov, V. P. Water and Monovalent Ions in the Minor Groove of B-DNA Oligonucleotides as Seen by NMR. Biopolymers 1998, 48, 210-233.

18. Tippin, D. B.; Sundaralingam, M. Nine Polymorphic Crystal Structures of d(CCGGGCCGG), d(CCGGGCCm(5)CGG), $\mathrm{d}(\mathrm{Cm}$ (5(CGGGCCm(5)CGG), and d(CCGGGCC(Br)(5)CGG) in Three Different Conformations: Effects of Spermine Binding and Methylation on the Bending and Condensation of ADNA. J. Mol. Biol. 1997, 267, 1171-1185.

19. Egli, M.; Williams, L. D.; Gao, Q.; Rich, A. Structure of the Pure-Spermine Form of Z-DNA (Magnesium Free) at 1-A Resolution. Biochemistry 1991, 30, 11388-11402.

20. Tishchenko, E. I.; Karapetyan, A. T.; Borisova, O. F. Different Complexes of Ethidium Bromide and Their Role in Stabilizing the $(\mathrm{dA})_{\mathrm{n}} \cdot(\mathrm{dT})_{\mathrm{n}}$ Structure. Mol. Biol. 1996, 30, 826-829.

21. Vardevanyan, P. O.; Antonyan, A. P.; Manukyan, G. A.; Karapetyan, A. T.; Shchyolkina, A. K.; Borisova, O. F. Ethidium Bromide Binding to Native and Denatured Poly(dA)Poly(dT). Mol. Biol. 2000, 34, 272-276.

22. Bradbury, E. M. Nucleosome and Chromatin Structures and Functions. J. Cell. Biochem. 1998, 30/31, 177-184.

23. Blackburn, G. M.; Gait, M. J. Nucleic Acids in Chemistry and Biology; 2nd ed. Oxford University Press: New York, 1996.

24. Porschke, D. Nature of Protamine-DNA Complexes: A Special Type of Ligand Binding Cooperativity. J. Mol. Biol. 1991, 222, 423-433.

25. Ausió, J. Histone H1 and Evolution of Sperm Nuclear Basic Proteins. J. Biol. Chem. 1999, 274, 31115-31118.

26. Tang, X.; Callahan, J. H.; Zhou, P.; Vertes, A. Noncovalent Portein-Oligonucleotide Interactions Monitored by MatrixAssisted Laser Desorption/Ionization Mass Spectrometry. Anal. Chem. 1995, 67, 4542-4548.

27. Karas, M.; Hillenkamp, F. Laser Desorption Ionization of Proteins with Molecular Masses Exceeding 10,000 Daltons. Anal. Chem. 1988, 60, 2299-2301.

28. Schildkraut, C.; Lifson, S. Dependence of the Melting Temperature of DNA on Salt Concentration. Biopolymers 1965, 3, 195-208.

29. Lin, S.; Cotter, R. J.; Woods, A. S. Detection of Non-Covalent Interaction of Single and Double Stranded DNA with Peptides by MALDI-TOF. Proteins 1998, S2, 12-21.

30. Harrison, J. G.; Balasubramanian, S. Synthesis and Hybridization Analysis of a Small Library of Peptide-Oligonucleotide Complexes. Nucleic Acids Res. 1998, 26, 3136-3145.

31. Robledo-Luiggi, C.; Vera, M.; Cobo, L.; Jaime, E.; Martinez, C.; Gonzalez, J. Partial Intercalation with Nucleic Acids of Peptides Containing Aromatic and Basic Amino Acids. Biospectroscopy 1999, 5, 313-322. 